\title{
Drosophila pattern formation: a meeting review
}

With the picturesque Flatirons as a backdrop, the molecular and developmental genetics of Drosophila pattern formation were discussed at the University of Colorado, Boulder (January 7-10, 1988). Here I wish to review selected aspects of the meeting, focusing on two main areas (and noting that what is omitted is not unimpor$\tan t)$ : first, the systems in the egg that generate the spatial cues for embryonic development and, second, the prospects for linking the outcome of these spatial cues to cell behavior in the embryo.

\section{Building a system into the egg that generates spatial information}

The fruit fly embryo is organized into repeating units (segments) along the antero-posterior (A-P) axis, and also exhibits polarity about the dorso-ventral (D-V) axis. For some time, it has been recognized that the spatial cues for generating this pattern must be contained within the fertilized egg. Elegant experimental manipulation of the insect egg and its contents by Sander (1976), Yajima (1960), and Kalthoff (1979) showed that there are two 'activity centers', one at the anterior pole and one at the posterior pole of the embryo, that exert long-range and graded effects on A-P patterning. Several talks made clear that we are on the verge of understanding what comprises activity centers such as these, and how they work in molecular terms.

During oogenesis, 15 nurse cells feed the growing oocyte through cytoplasmic bridges. These germ-line cells (oocyte and its sister nurse cells) are surrounded by somatically derived follicle cells, which build the egg's protective vitelline membrane and shell (chorion). The shape of the chorion, like that of the egg, has a pronounced $\mathrm{D}-\mathrm{V}$ and $\mathrm{A}-\mathrm{P}$ asymmetry.

Among the genes that must be active in the mother during the formation of the egg, two classes are involved in establishing the anterior and posterior pole activity centers described by classical manipulation experiments. Two other classes generate $\mathrm{D}-\mathrm{V}$ polarity: one class affects both egg shell and embryo, while a lateracting class affects only the embryo.

\section{The $\mathbf{D}-\mathrm{V}$ axis}

\section{Cooperation between germ line and somatic cells}

Results reported by Schüpbach (Princeton University) indicate that the somatic follicle cells and the germ line communicate to organize correctly the $\mathrm{D}-\mathrm{V}$ axis of the egg and embryo. Several genes are involved in patterning both the egg shell and the embryo. Mutations in two genes, gurken and torpedo, cause a ventralization of the egg shell and of the resulting embryo (Schüpbach 1987).

\footnotetext{
*When discussing such 'maternal effect' genes, 'mutant embryos' refers
} to embryos from homozygous mutant mothers.
Mutations in two other genes, spire and cappuccino, cause a variable dorsalization of egg and embryo, along with more constant abdominal and pole cell defects. Schupbach has determined where the activity of these genes is needed by building 'mosaic' females with a mutant germ line (nurse cells and oocyte) and wild-type somatic cells (follicle cells), or vice versa. A mutant gurken, spire, or cappuccino germ line results in a defective egg shell, even if the shell is produced by wild-type follicle cells. This implies that the follicle cells (soma) derive information to shape the egg shell from genes expressed in the germ line. Reciprocally, torpedo activity is required in the soma. In this case a mutant soma results not only in a defective egg shell but also in a mutant embryo. This implies that the pattern-generating system built into the egg by the germ line (nurse cells) is influenced by the surrounding somatic cells (most likely the follicular epithelium).

The cellular basis for this interaction will be interesting since, in addition to alleles that affect the follicular epithelium (maternal effect alleles), zygotic lethal alleles of torpedo have been identified that exhibit effects on other single cell layer epithelia. Perhaps torpedo activity, Schupbach surmises, is central to the construction and arrangement of epithelia in general.

\section{The next class of $D$ - $V$ genes}

Genetic analyses suggest that the next step in D-V patterning involves genes whose requirement is restricted to the germ line, only affecting the resulting embryo and not the chorion. Two members (Toll and easter) of this class can be mutated both to recessive, dorsalizing alleles and to dominant, ventralizing alleles (Anderson, University of California, Berkeley). Genetic studies suggest that the dominant alleles are not due to reduced gene activity, nor to overproduction of a wild-type product. In addition, injection of $\mathrm{Toll}^{+}$cytoplasm into Toll mutants creates a D-V axis with a ventral focus at the site of injection. Curiously, both rescuing activity and Toll RNA are homogeneously distributed in the early embryo. Thus, precisely what constitutes the asymmetry and drives ventralization in these cases is the central (and open) question. By analyzing mutant combinations of easter and Toll, Anderson concludes that easter acts prior to Toll (e.g., easter ${ }^{\text {dorsal }}$, Toll ventral yields a ventralized embryo). The molecular and biochemical analyses of these products are required to determine if easter activity may modifies Toll activity.

Toll is also expressed later in embryogenesis by the zygotic genome, and is localized in areas undergoing cell movements: invaginating cells that make tracheal pits and salivary glands; cells apposed at ventral furrow closure; and muscle-epithelia attachment sites. This localization may imply that Toll acts in cell-cell association. 
Molecular analysis of Toll does indeed point in this direction. The predicted protein has the hallmarks of a transmembrane protein (putative signal sequence, sites for $N$-linked glycosylation, a membrane-spanning domain; Hashimoto et al. 1988). Additionally, it contains a leucine-rich repeat like that found in a human serum protein (LRG) (Takahashi et al. 1985), the receptor for von Willebrand factor (Lopez et al. 1987), adenylate cyclase of yeast (Reinke et al. 1988), and the Drosophila photoreceptor glycoprotein chaoptin (Reinke et al. 1988). Putnam has proposed that this repeat may provide a capacity for association with membranes (Takahashi et al. 1985). The areas of zygotic Toll expression are consistent with such a role. The situation for maternal Toll is less clear. Since the D-V axis is being specified during the early and syncytial phase of embryogenesis, to what membranes might the Toll product be localized or with what membranes might it be associated?

\section{Polar activity centers}

\section{Anterior activity}

Driever (Max-Planck-Institut) presented an elegant analysis of a morphogenetic protein gradient that is likely to be the major component of the anterior activity center. Activity of the maternally expressed gene bicoid is required for development of anterior structures in the embryo. The RNA encoded by bicoid is produced by nurse cells and deposited in the growing oocyte, where it is localized to the anterior-most cytoplasm. Two genetic functions, exuperentia and swallow, appear responsible for this anterior localization (Frohnhöfer and NüssleinVolhard 1987). Using antibodies to the bicoid protein, Driever reported that this product quickly forms a steep concentration gradient over the anterior two-thirds of the embryo after fertilization. The shape of the protein gradient (an exponential drop of approximately two orders of magnitude) might be determined by the rate of localized synthesis, the rate of 'diffusion' away from the source RNA, and the rate of degradation.

Altering the gene dose of bicoid ${ }^{+}$alters the gradient's shape, and such alterations of the gradient have effects on development of the body pattern. For example, cells at a particular position in the embryo invaginate at the onset of gastrulation, producing a 'head fold'. With a change in the shape of the bicoid gradient, there is a parallel change in the position of this head fold. Raising the dose of bicoid ${ }^{+}$shifts both the head fold and the protein gradient to the posterior, while lowering bicoid gene dose shifts both anteriorly. This confirms the central role played by the bicoid product in the determination of anterior structures. However, the magnitude of the head fold shift is less than the magnitude of the shift in absolute bicoid protein concentration. This implies that it is not the absolute level of bicoid protein that determines head fold position directly, but rather that bicoid acts via other factors, most likely the sequentially activated zygotic segmentation genes.

\section{Posterior activity}

Two talks (Boswell, University of Colorado, Boulder; Lehmann, MRCl focused on mutants of seven maternally acting genes that affect the development and po- larity of the posterior region (abdominal phenotype). Mutations in five of these genes are also defective for the specialized posterior pole cytoplasm required for the production of pole cells (pole cell phenotype). It is useful to determine whether the components identified by these seven genes work independently, and to assign certain gene products to particular steps.

Wild-type posterior pole plasm, when injected into mutant embryos, can rescue the abdominal and pole cell phenotypes, whereas injecting more anteriorly located wild-type cytoplasm cannot rescue. This shows that rescue activity is localized spatially to the posterior pole. The abdominal phenotype can be rescued by injection of wild-type pole plasm into the abdominal region of mutants, showing that one focus of action for the polar activity is in the prospective abdominal region. Finally, mutations in gene ' $a$ ' cannot rescue mutations in gene ' $b$ ', indicating that the different loci do not act independently. The interesting exception to this is $p u$ milio pole plasm, which not only rescues the other mutants, but also rescues the abdominal defects of pumilio embryos if injected into the prospective abdominal region (Lehmann and Nüsslein-Volhard 1987). Therefore, pumilio mutants produce an activity center and lead Lehmann to conclude that pumilio mutants lack the capacity to 'transport' the active material from the pole plasm (the 'source') to the site of action (the abdomen). Further transplantation tests should fine-tune a working model for the other six genes.

\section{From 'morphogen' to effector}

The spatial information established by the action of these maternally acting loci becomes progressively refined through the early stages of embryogenesis, culminating in a richness of pattern by the cellular blastoderm. This refinement involves the sequential activation, by the maternal activities, of a hierarchy of zygotically acting genes. One major (and elusive) goal in the field is to understand how these zygotically acting genes actually affect cell behaviors. Two reports establish what may be the groundwork for doing just that.

\section{Intricate patterns of cell cycle times at cellular blastoderm}

Whereas nuclei divide with near synchrony during the first 13 mitotic divisions in the syncitial embryo, this synchrony is lost after the establishment of the cellular blastoderm and replaced with a beautifully patterned mitotic program. Foe (Friday Harbor Labs, University of Washington/ has mapped some 25 -odd mitotic domains (groups of meta-synchronously dividing cells), building upon earlier work by Poulson (1950), Turner and Mahowald (1977) and Hartenstein and Campos-Ortega (1985). Cells making up a domain share, by definition, a common cell cycle duration. Additionally, in some domains the cells exhibit unique properties distinct from cells in adjacent domains, including cell shape, mitotic spindle orientation, and gene expression programs. Foe suggests that the cells comprising each domain share a genetic commitment.

The complexity (and precision) manifested in the appearance of the mitotic domains attests to the intricacy of the genetic patterning carried out by the earlier-acting 
regulatory network. Perhaps we are ready to ask how particular genes within this network act upon the circuitry controlling the cell cycle.

\section{Genetics of cellularization and gastrulation}

The other obvious events at the cellular blastoderm are the act of cellularization itself and the onset of the morphogenetic cell shape changes, gastrulation.

Wieschaus and colleagues have characterized several regions of the Drosophila genome that are required for events that occur at the time of cellularization. Two regions are required for cellularization itself: One region blocks an early slow phase, which involves new membrane synthesis, and the second region blocks a later, contractile phase, which pulls the newly added membranes down around each nucleus. If the slow phase is blocked, the fast phase still takes place at the correct time, indicating that these phases are independent of one another (what is the clock?). Two other regions affect the fidelity of the cellularization process. In one region, deficiencies cause both the encapsulation of several nuclei into a single cell and the formation of cells having no nuclei. The fidelity of cellularization may involve some interplay between the nuclei and cortically located actin caps. Are functions encoded within this deficiency involved in the organization of actin cytoskeleton? During cellularization, basally located cytoplasm optically clears as vesicles and lipid droplets are transported inward to the yolky milieu. Deficiency of another region affects this clearing by blocking the inward transport of lipid droplets.

Gastrulation involves several concerted cell-group movements (ventral furrow, cephalic furrow), cell shape changes (anterior and posterior midgut cell plate formation), and the onset of germband elongation, a process in which cells slip past one another as the D-V axis compresses and the embryo elongates along the A-P axis. What mechanisms drive these movements?

Wieschaus has identified about a dozen zygotically required gene activities that affect gastrulation by blocking region-specific changes in cell behavior. His group has begun to examine several of these in detail. For example, the characteristic change in shape of cells located at the posterior pole does not occur in folded gastrulation mutants. By using mosaic analysis, it has been shown that the activity of three out of four of these gastrulation-defective genes is required locally, generally correlating with cells affected in the mutant.

In principle these mutations may interfere with the cellular apparatus that produces the shape change, or cause the cells to assume the wrong positional identity and adopt the behavior of other cells. Wieschaus has begun to distinguish among these possibilities for eight of the mutants by scoring an independent marker for cell position (Foe's cells cycle timing). Four mutants show a normal mitotic pattern and, thus, may block the execution of the cell behavior. Four mutants exhibit an altered mitotic program, suggesting that the cells may have assumed the wrong fate.

Cell shape changes must involve the cytoarchitecture of cells. Cytoskeletal components, their interacting proteins, and their dynamics are under intense investigation in many systems. Perhaps analysis of gastrulationdefective mutants can complement these analyses.
These cellular aspects of development /control over cell cycle length, the process of cellularization, the morphogenetic movements of gastrulation) are interesting in their own right. Additionally, being placed as they are at the transition between a regulatory hierarchy and changes in cellular behavior, the components that regulate these processes may be the link between regulatory factors that determine a cell's fate and the effector molecules that execute the behavior of a cell /which is that cell's fate). The message was clear from Boulder: The year ahead should be illuminating

\section{Stephen DiNardo}

Department of Biochemistry and Biophysics

University of California, San Francisco

San Francisco, California 94143

\section{References}

Frohnhöfer, H.G. and C. Nüsslein-Volhard. 1987. Maternal genes required for the anterior localization of bicoid activity in the embryo of Drosophila. Genes Dev. 1: 880-890.

Hartenstein, V. and J.A. Campos-Ortega. 1985. Fate-mapping in wild-type Drosophila melanogaster. I. The spatio-temporal pattern of embryonic cell divisions. Wilhelm Roux's Arch. Dev. Biol. 194: 181-195.

Hashimoto, C., K.L. Hudson, and K.V. Anderson. 1988. The Toll gene of Drosophila, required for dorsal-ventral polarity, appears to encode a transmembrane protein. Cell 52: 269279.

Kalthoff, K. 1979. Analysis of a morphogenetic determinant in an insect embryo. In Determinants of spatial organisation (ed. S. Subtelney and I. Konigsberg), pp. 97-126. Academic Press, New York.

Kataoka, T., D. Broek, and M. Wigler. 1985. DNA sequence and characterization of the $S$. cerevisiae gene encoding adenylate cyclase. Cell 43: 493-505.

Lehmann, R. and C. Nüsslein-Volhard. 1987. Involvement of the pumilio gene in the transport of an abdominal signal in the Drosophila embryo. Nature 329: 167-170.

Lopez, J.A., D.W. Chung, K. Fujikawa, F.S. Hagen, T. Papayannopoulou, and G.J. Roth. 1987. Cloning of the chain of human platelet glycoprotein lb: a transmembrane protein with homology to leucine-rich $\alpha_{2}$-glycoprotein. Proc. Natl. Acad. Sci. 84: 5615-5619.

Poulson, D.F. 1950. Histogenesis, organogenesis, and differentiation in the embryo of Drosophila melanogaster Meigen. In Biology of Drosophila (ed. M. Demerec), pp. 168-274. John Wiley, New York.

Reinke, R., D.E. Krantz, D. Yen, and S.L. Zipursky. 1988 Chaoptin, a cell surface glycoprotein required for Drosophila photoreceptor cell morphogenesis, contains a repeat motif found in yeast and human. Cell 52: 291-301.

Sander, K. 1976. Specification of the basic body pattern in in sect embryogenesis. Adv. Insect Physiol. 12: 125-238.

Schüpbach, T. 1987. Germ line and Soma cooperate during oogenesis to establish the dorsoventral pattern of egg shell and embryo in Drosophila melanogaster. Cell 49: 699-707.

Takahashi, N., Y. Takahashi, and F.W. Putnam. 1985. Periodicity of leucine and tandem repetition of a 24-amino acid segment in the primary structure of leucine-rich $\alpha_{2}$-glycoprotein of human serum. Proc. Natl. Acad. Sci. 82: 1906 1910.

Turner, F.R. and A.P. Mahowald. 1977. Scanning electron microscopy of Drosophila melanogaster embryogenesis. II. Gastrulation and segmentation. Dev. Biol. 57: 403-416.

Yajima, H. 1960. Studies on embryonic determination of the harlequin-fly, Chironomus dorsalis. I. Effects of centrifugation and of the combination with constriction and puncturing. J. Embryol. Exp. Morphol. 8: 198-215. 


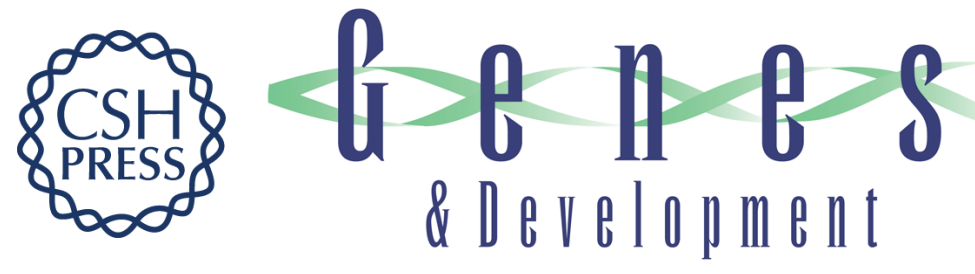

\section{Drosophila pattern formation: a meeting review}

Genes Dev. 1988, 2:

Access the most recent version at doi:10.1101/gad.2.6.617

References This article cites 12 articles, 3 of which can be accessed free at: http://genesdev.cshlp.org/content/2/6/617.full.html\#ref-list-1

License

Email Alerting

Service

Receive free email alerts when new articles cite this article - sign up in the box at the top right corner of the article or click here.

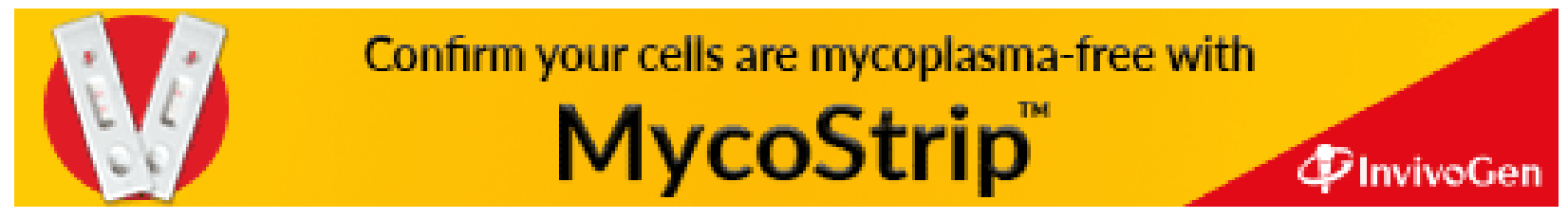

